\title{
Pagsipat sa mga salik na nakaimpluwensiya sa varayti at varyasyong leksikal sa wikang Chabacano ng lungsod Zamboanga
}

Drapeza, Hanna $\measuredangle$

Western Mindanao State University, Philippines (drapeza.hanna@wmsu.edu.ph)

Reyes, Aubrey

Western Mindanao State University, Philippines (aubreyfreyes80@gmail.com)

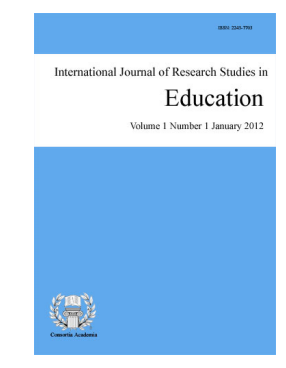

ISSN: 2243-7703 Online ISSN: 2243-7711

OPEN ACCESS

\section{Abstract}

This study has revealed that nouns, adjectives and verbs are partially the same in both rural and urban areas. However, there are few words in Chabacano language that differs in terms of usage in both areas. Moreover, the study has shown that migration due to education, marriage/cross marriage and employment, influence of media (television and radio) and social media are the factors that influenced the variety and lexical variation in Chabacano language in rural and urban areas in Zamboanga City. Also, emigrants could influence the surroundings where informants reside. Lastly, this study revealed that Spanish language has the largest influence in Chabacano language. Based on the findings of this study, the following conclusions were formed: that Chabacano language is just like other languages in the Philippines which are subjected to variations such as Filipino language. Language variations which are featured are results of demographic factors such as geographical differences of speakers, although some words still remain in rural and urban areas, the data shows that more lexical words remain concentrated in the rural area of Zamboanga City. The existence of variation in Chabacano language results in having many languages spoken in their respective places which have a great influence on the fact that they have different words that sometimes come from other languages spoken by other communities. The use of other languages due to dwelling of other tribes is a major contributor to the variety and lexical variation of the Chabacano language.

Keywords: variation; lexical items; lexicon; Chabacano; rural barangay; urban barangay 


\section{Pagsipat sa mga salik na nakaimpluwensiya sa varayti at varyasyong leksikal sa wikang Chabacano ng lungsod Zamboanga}

\section{Introduksiyon}

Nakikilala ang tao kung saan at ano ang kinabibilangang grupo, trabaho, pook na tinitirhan, edukasyon, kultura, lahi, at relihiyon sa pamamagitan ng wikang ginagamit. Magkatulad man ang tawag o pangalan ng wika sa isang munisipalidad, barangay, o sitio na nabibilang sa isang pook o lalawigan ay nagkakaroon pa rin ito ng pagkakaiba na maaaring sa bokabularyo, tunog at/o gramatika. Dito lumitaw ang varayti ng isang wika. Katulad na lamang ng wikang Chabacano na kakikitaan ng iba't ibang varayti at varyasyon. Ayon kay Austero (2007), ang varayti ay bunga ng varyasyon na maaaring nasa tunog, mga salita o bokabularyo at sa istrukturang gramatika. Nagaganap din ito ayon sa mga sosyolingguwistiko bunga ng konteksto ng etnisiti, sosyal, klas, seks, heyograpiya, edad at ibang paktor.

Chabacano ang lingua franca ng humigit kumulang limang daang libong (500,000) Zamboangueño ng Lungsod Zamboanga. Naging tanyag ang Zamboanga, bilang Asia's Latin City sa buong Asya dahil ang wikang Chabacano ay may pagka-Español. Ang Zamboanga ay isang lungsod ng rehiyon na tangway ng Pilipinas. Ito ay may kabuoang land area na 1,483.38 sq.km. Ikatlong pinakamalaking lungsod ng Pilipinas sa lupain at may kabuuang 98 barangay na nahahati sa rural at urban barangay ng lungsod. Ang mga barangay na kabilang sa rural ay mga taal na Zamboangueño, sila ay matagal nang nanirahan sa lugar na ito at nagsasalita ng purong Chabacano. Ibig sabihin hindi sila naimpluwensyahan ng ibang wika mula sa ibang mga karatig na lungsod kagaya ng Zamboanga del Norte at Zamboanga del Sur. Samantalang ang mga barangay na kabilang sa urban ay karaniwang nakikisalamuha sa iba't ibang lahi tulad ng mga Bisaya, Tagalog, Tausug, Subanen, at iba pa sapagkat dito kadalasang pumupunta at naninirahan ang mga dumadayo sa lungsod. Sa pagdating ng ibang mga lahi na tumira dito sa lungsod ng Zamboanga partikular sa urban barangays, hindi maiiwasang naiimpluwensiyahan ng mga grupong ito ng mga nakapaligid sa kanila sa aspektong leksikal.

Ang Chabacano ang ginagamit sa pagsasalita ng halos lahat ng mga tao sa lungsod ng Zamboanga, sa palengke, mga malalaking establisimiyento, mapaanong uri ng kabahayan, mga opisina at mga kainan ang ginagamit bilang midyum sa pakikipagtalastasan. Mapapansin na iba-iba ang mga katawagang ginagamit ng mga tao partikular na sa rural at urban barangay sa lungsod. Kadalasan, iba sa karaniwang katawagan ng wikang Chabacano ang ginagamit ng mga tao sa lungsod kung ikukumpara sa rural barangay. Nagkakaroon ng kaibahan ang paraan ng pagbigkas, maging sa katawagan nito.

Sa kasalukuyan, buhay na buhay pa rin ang Lingua Franca na Chabacano sa lungsod ng Zamboanga. May mga taal na Chabacano na naninirahan sa urban at rural. Sa mga lugar na tinitirhan ng mga Zamboangueños maririnig natin ang iba't ibang katawagan sa isang partikular na salita. Dahil nga sa iba't ibang katawagang ito ay nalilito at nagtataka ang ilan sa kung ano nga ba ang naaayon at nararapat. Mababakas din sa kanilang mga mukha ang pagkabahala sa sitwasyong pangwikang ito lalo na sa pakikipagtalastasan sa urban man o rural na mga barangay. Makikita rin sa kanilang mga reaksyon ang pagnanais na matukoy ang mga dahilan ng pagbabago sa wikang Chabacano.

\section{Metodolohiya}

Ang pag-aaral na ito ay isang kwalitatibong pananaliksik. Ginamit ang palarawang pamamaraan ng pagsusuri. Ginamit ng mananaliksik ang convenient sampling at snow ball technique. Tatlumpu (30) ang kabuoang bilang ng impormanteng ginamit sa pag-aaral na ito. Labinlima (15) sa urban, na pinangkat ayon sa distritong kinabibilangan. Ibig sabihin sila ay nakatira sa loob $\mathrm{ng} 7 \mathrm{~km}$ radius mula sa City Hall ng lungsod at labinlima (15) ring impormante sa rural barangay na pinangkat din ayon sa distritong kinabibilangan nito kung 
Pagsipat sa mga salik na nakaimpluwensiya sa varayti at varyasyong leksikal sa wikang Chabacano

saan ay nakatira sa labas ng $7 \mathrm{~km}$ radius ng lungsod ng Zamboanga. Ang instrumentong ginamit sa pagtataya ng pag-aaral na ito ay ang instrumentong ginamit ni Reyes (2015) sa kanyang konseptong papel na pinamagatang "Varayti at Varyasyon ng Wikang Chabacano sa Rural at Urban Area ng Lungsod ng Zamboanga". Ang talatanungan ay binubuo ng tatlong (3) set na batay sa bahagi ng pananalita. Mayroong labinlimang (15) salita sa pangngalan, labinlimang (15) salita sa pang-uri at labinlimang (15) salita sa pandiwa. Ito ay nakasulat sa wikang Filipino at tinumbasan sa wikang Chabacano batay sa kung paano nila ito ginagamit sa pang-araw-araw na pakikipagtalastasan at upang makuha ng mga mananaliksik ang pagpapakahulugan, interpretasyon at kaalaman ng mga Zamboangueño tungkol sa mga posibleng dahilan ng mga nagaganap na pagbabago sa wikang Chabacano ng rural at urban barangay ng lungsod ng Zamboanga.

Matapos makatanggap ng ethics clearance mula sa Research Ethics and Oversight Committee (REOC) ng Western Mindanao State University para sa implimentasyon, sinimulan na ang paglikom ng datos. Nagpadala ng liham pahintulot ang mananaliksik sa mga impormate ng ginawang pag-aaral. Kalakip sa liham ang paglalahad ng kaligiran, layunin, at kahalagahan ng ginawang pananaliksik. Tinalakay din sa liham, ang mga karapatan at responsibilidad ng mga impormante gaya ng karapatang maitago ang kanilang totoong identidad, hindi makibahagi kung sa tingin nila ay hindi makatutulong sa anumang paraan ang paglahok sa pag-aaral at ang karapatang umatras anumang oras sa panahon ng pangangalap ng datos. Tiniyak ng mga mananaliksik ang kaligtasan at kaayusan ng mga impormante sa buong panahon ng paglilikom ng datos. Nagsagawa ng pakikipanayam ang mga mananaliksik mula sa mga impormanteng ito. Ginamit ng mga mananalisik ang paraang pagtatanong-tanong. Ayon kay Gonzales (1976) mula kay Yabut (2003) sa isang pagtatanong-tanong, walang itinakdang estruktura sa mga tanong at ang pinakamahalagang gabay ng mananaliksik ay ang hangarin ng pananaliksik. Nilalayon ng pagtatanong-tanong ang mabigyang-linaw ang mga salik na nakaimpluwensya sa pagkakaroon ng varayti at varyasyong leksikal sa wikang Chabacano ng lungsod Zamboanga. Ang pangangalap ng datos ay pahirapan dahil ang panayam sa mga impormante ay isinagawa sa pamamagitan lamang ng telepono batay na rin sa pagsunod sa pamantayang ibinigay ng IATF. May mga panahong hindi maayos ang signal ng TelCo. (Telecommunication) kaya paulit-ulit ang pagtawag sa mga impormante na siyang nagdulot ng matagal na paglikom ng mga datos. Ito ang naging limitasyon ng pag-aaral.

\section{Paglalahad ng resulta}

Ang seksyon na ito ay naglalahad, nagsusuri at nagbibigay ng mga datos na nakalap upang maipakita ang iba't ibang salik na nakaimpluwensiya sa pagkakaroon ng baryasyong leksikal ng wikang Chabacano sa rural at urban area ng lungsod ng Zamboanga.

\section{Talahanayan 1}

Leksikal na pagkakaiba ng wikang Chabacano sa rural at urban barangay

\begin{tabular}{|c|c|c|}
\hline $\begin{array}{lr} & \text { Filipino } \\
\text { Pangngalan }\end{array}$ & $\begin{aligned} \text { Chabacano Rural } \\
\end{aligned}$ & Chabacano Urban \\
\hline \\
\hline \multicolumn{3}{|c|}{ Biriha } \\
\hline \multirow{4}{*}{ Hita } & \multirow{3}{*}{$\begin{array}{l}\text { Pierna } \\
\text { Hita }\end{array}$} & \multirow{2}{*}{$\begin{array}{c}\text { Biriha } \\
\text { Pierna } \\
\text { Hita }\end{array}$} \\
\hline & & \\
\hline & & Empeinera \\
\hline & \multirow{2}{*}{$\begin{array}{l}\text { Garrote } \\
\text { Viyucu } \\
\text { Palo } \\
\text { Latigo }\end{array}$} & \multirow{2}{*}{$\begin{array}{l}\text { Garrote } \\
\text { Viyucu }\end{array}$} \\
\hline \multirow{3}{*}{ Garote } & & \\
\hline & \multirow{2}{*}{\multicolumn{2}{|c|}{ the }} \\
\hline & & \\
\hline Luha & Lagrimas & \multirow{2}{*}{$\begin{array}{l}\text { Lagrimas } \\
\text { Luha } \\
\text { Rodillas } \\
\text { Tuhod }\end{array}$} \\
\hline Tuhod & Rodillas & \\
\hline
\end{tabular}

Nota. Matutunghayan sa talahanayan 1.0 ang mga panumbas na salita sa Chabacano sa bahagi ng pananalitang Pangngalan. 
Drapeza, H., \& Reyes, A.

\section{Talahanayan 2}

Leksikal na pagkakaiba ng wikang Chabacano sa rural at urban barangay

\begin{tabular}{|c|c|c|}
\hline Filipino & Chabacano Rural & Chabacano Urban \\
\hline \multicolumn{3}{|l|}{ Pang-uri } \\
\hline \multirow[t]{3}{*}{ Luma } & Daan & Daan \\
\hline & Antiguo & \\
\hline & De antes & \\
\hline \multirow[t]{3}{*}{ Maganda } & Bonita & Bonita \\
\hline & Hermosa & Hermosa \\
\hline & Buenmoso & \\
\hline \multirow[t]{4}{*}{ Madamot } & Canalla & Canalla \\
\hline & Lagac & Lagac \\
\hline & Curipot & Curipot \\
\hline & Mutui hambre & \\
\hline \multirow[t]{4}{*}{ Guwapo } & Hermusura & Hermusura \\
\hline & Hermoso & Hermoso \\
\hline & Guapo & Guapo \\
\hline & Plantudo & \\
\hline \multirow[t]{5}{*}{ Mabilis } & Lijierro & Lijierro \\
\hline & Pronto & Pronto \\
\hline & Corre & Corre \\
\hline & Paspas & Paspas \\
\hline & & Fuerte \\
\hline \multirow[t]{3}{*}{ Sinungaling } & Embustero & Embustero \\
\hline & Pamparon & Pamparon \\
\hline & & Imbusterias \\
\hline
\end{tabular}

Nota. Matutunghayan sa talahanayan 1.0 ang mga panumbas na salita sa Chabacano sa bahagi ng pananalitang Pang-uri.

\section{Talahanayan 3}

Leksikal na pagkakatulad ng wikang Chabacano sa rural at urban barangay

\begin{tabular}{|c|c|c|}
\hline Filipino & Chabacano Rural & Chabacano Urban \\
\hline \multicolumn{3}{|l|}{ Pandiwa } \\
\hline \multirow[t]{3}{*}{ Nagmadali } & Ya apurra & Ya apurra \\
\hline & Apurrao & Apurrao \\
\hline & Pronto & \\
\hline \multirow[t]{3}{*}{ Tinanggal } & Ya quita & Ya quita \\
\hline & Destronca & Ya destronca \\
\hline & Despone & Ya arranca \\
\hline \multirow[t]{3}{*}{ Nagsisi } & Ya arripinti & Ya arripinti \\
\hline & Aripintido & Yan mahay \\
\hline & Arripinti & Yan sisi \\
\hline \multirow[t]{2}{*}{ Tumatakas } & Ta escapa & Ta escapa \\
\hline & Ta desalena & \\
\hline \multirow[t]{4}{*}{ Bubugbugin } & Ay garrucha & Ay garrucha \\
\hline & Ay golpea & Golpea \\
\hline & Ay Bobotia & Ta embona \\
\hline & & Ta baketcha \\
\hline
\end{tabular}

Nota. Matutunghayan sa talahanayan 1.0 ang mga panumbas na salita sa Chabacano sa bahagi ng pananalitang Pandiwa.

\section{Diskusyon}

Mapapansin mula sa talahanayan 1.0 na ang mga salitang Pangngalan sa Filipino ay may mga salitang panumbas sa rural na wala sa urban. Ang Pangngalan ay bahagi ng pananalita na tumutukoy sa ngalan ng tao, bagay, hayop, lugar at pangyayari. Tulad ng salitang palo at latigo na katumbas ng garote sa Filipino na ang ibig sabihin ay bagay na ginagamit na pamalo. Ang salitang empeinera ay may katumbas sa Filipino na hita. Sa 
Chabacano ang empeinera ay nangangahulugang dalawang hita subalit ang empeinera ay salitang Español na nangangahulugang "I started". Samantala ang mga salitang luha at tuhod ay mga salita na matatagpuan sa urban na wala naman sa rural. Ang mga salitang luha at tuhod ay salitang Bisaya at Tagalog.

Makikita naman sa talahanayan 2.0 na ang mga salitang Pang-uri sa Filipino ay may mga salitang panumbas sa rural na wala sa urban. Ang Pang-uri sa bahagi ng pananalita ay naglalarawan sa pangngalan at panghalip tulad ng mga salitang antiguo/de antes (luma), buenmoso (maganda), mutui hambre (madamot), plantudo (guwapo), at mal echos/tecasin/embustero (manloloko) ay ginagamit sa rural area habang ang salitang fuerte (mabilis), embusterias (sinungaling), at traydor ay ginagamit sa urban area. Mapapansin na ang mga salitang Pang-uri sa rural at urban area ay mga orihinal na katawagang Chabacano.

Makikita rin sa talahanayan 3.0 na ang mga salitang Pandiwa na sa bahagi ng pananalita ay nagsasaad ng kilos gaya ng pronto, ta desalena, at garrucha ay mga salitang rural na wala sa urban samantala ang salitang ta baketcha ay salitang urban na wala naman sa rural area. May mga salitang magkaiba ang katawagan sa rural at urban katulad na lamang ng salitang tinanggal na may katumbas na katawagan sa rural na despone habang sa urban ay ya arranca. Ito ay mga orihinal na katawagang Chabacano. Ang mga salitang nagsisi na may katumbas na katawagan sa rural na aripintido at arripinti habang sa urban naman ay yan mahay at yan sisi ay nagpapahiwatig na ang mga salitang nasa urban ay impluwensiya ng iba pang wika. Ang mahay ang salitang mula sa Bisaya habang ang sisi ay mula sa salitang Tagalog. Isa pang halimbawa ang salitang bugbugin na may katumbas na ay bobotia sa rural habang ta embona naman sa urban. Ang mga ito ay mga orihinal ring salitang Chabacano.

Batay sa mga datos na nalikom, ang mga salita ay may pagkakaiba sa rural at urban barangay sa bahagi ng pananalita na Pangngalan, Pang-uri at Pandiwa. Makikita sa mga datos na ang bawat lugar sa lungsod ng Zamboanga na gumagamit ng wikang Chabacano ay may varyasyon. Ito'y nagpapatunay sa konsepto ni Arbes (2010) mula sa aklat nina Santos et al. (2010) na ang pagkakaiba-iba ng mga panumbas sa iisang salita at paraan ng pagkakabuo ng mga salitang ito ay nagpapatunay lamang na ang wika ay hindi homogeneous. Dagdag pa nina Paz et al. (2003), hindi homogeneous ang isang wika dahil may iba-iba itong anyo. Dahil sa tuloy-tuloy na interaksyon at pakikipag-usap ng mga miyembro ng isang grupo o komunidad tulad ng pamilya, mga kaparehong propesyon at iba pa nagkakaroon ng mga katangian ang mga salita nila na naiiba sa mga miyembro ng ibang grupo o komunidad. Napatunayan rin ang sinabi ni Rubico (2009) na ang varyasyon ay makikita sa leksikon at paraan ng pagbubuo o anyo ng salita. Napatunayan din sa lumabas na datos ng pag-aaral ang teoryang sosyolingguwistiko ni Saussure (1915), makikita ang paghahalo ng mga barayti ng wika, diyalekto at register. Tulad ng mga salitang ay bobotia /ay embona / ay baketia (bubugbugin) na mga salitang mula sa iba't ibang varayti ng wikang Chabacano na ginagamit sa rural at urban barangay sa lungsod ng Zamboanga. Sa paraang ito, ang isang salita o higit pa ay ginagamit mula sa isang varayti tungo sa isa pang varayti dahil ito ang nakasanayang ginagamit ng nagsasalita.

Batay sa datos na nakuha sa pag-aaral, isa sa mga posibleng dahilan ay ang paglipat ng tirahan ng mga tao mula sa rural patungong urban sa lungsod ng Zamboanga. Tulad lamang ng salitang Pangngalan na hita na may katumbas na empeinera sa urban. Ito'y salitang Chabacano na tinumbasan ng impormante mula sa urban subalit walang nagbigay ng katumbas nito sa rural barangay. Maraming mamamayan sa rural barangay na piniling bumaba o manirahan sa urban barangay upang mag-aral, magtrabaho o dili kaya'y nakahanap ng kabiyak na iba ang unang wikang ginagamit. Kung kaya ang mga salitang Chabacano ay napapalitan ng ibang katawagan na madaling maunawaan at kadalasang ginagamit ng mga tao sa urban barangay. Dahil sa patuloy na paggamit nito, dahan-dahan ng nakasanayan at napapalitan ang salita hanggang sa naipapasa rin ito sa kanilang mga anak at naging bahagi na ng kanilang unang wika. Maging ang mga kabataan ngayon ay siyang pinanggagalingan ng mga pagbabagong nagaganap sa wikang Chabacano dala ng malaimpluwensiyang paligid kung saan sila lumaki, nag-aral at maging sa kanilang nakakasalamuha sa araw-araw. Malaki rin ang kontribusyon ng midya hindi lamang sa kabataan maging sa lahat na nanonood o gumagamit nito (telebisyon, radyo o social media) na naging dahilan ng paglapit ng mga tao sa makabagong henerasyon sanhi ng pagbabagong nagaganap sa unang wika. 
Batay rin sa naitalang sarbey ng bawat barangay na binisita ng mga mananaliksik, lumabas sa kanilang talaan na lumalaki ang bahagdan kada taon ng mga dayong mula sa iba't ibang bayang nagdisisyong manirahan sa kanilang pook na hindi maikaiila na may malaking kontribusyon sa pagbabagong nagaganap sa wikang Chabacano. Makikita sa Talahanaya 4 sa ibaba ang pangkalahatang sensus ng lungsod Zamboanga mula taong 1970 hanggang 2010. Ang kabuoang populasyon sa taong 1970 ay 199.9, taong 1980 ay populasyon bilang 434.7, taong 1990 naman ay 442.3 , taong 2000 ay 601.8 at sa taong 2010 ay may populasyong bilang na 807.1.

\section{Talahanayan 4}

Kabuoang Populasyon ng lungsod Zanboanga batay sa Census taong 1970-2010

\begin{tabular}{|cc|} 
Table 1 \\
\multicolumn{3}{|c|}{$\begin{array}{c}\text { Total Population by Census Year } \\
\text { Zamboanga City, 1970 to 2010 }\end{array}$} \\
\hline $\begin{array}{cc}\text { Decennial } \\
\text { Census }\end{array}$ & $\begin{array}{c}\text { Total Population } \\
\text { (in thousands) }\end{array}$ \\
\hline 1970 & 199.9 \\
1980 & 434.7 \\
1990 & 442.3 \\
2000 & 601.8 \\
2010 & 807.1 \\
\hline
\end{tabular}

Nota. Kinalap mula sa https://www.google.com/search?q=Zamboanga+City+sensus+2019

Halimbawa na lamang ang salitang aso na tinumbasan ng salitang iru sa urban. Mahihinuha na ang salitang iru ay mula sa salitang Bisaya o Tausug. Maraming kabahayan sa iilang pook ng urban barangay na pinalilibutan ng iba't ibang etnikong lahi partikular na sa mga barangay ng Baliwasan, Canelar, Tetuan, Putik, Tumaga Porcentro, Tugbungan, Mampang, Pasonanca, Sta. Maria at San Roque. Mula sa datos na nakalap ng mananaliksik, hindi maitatanggi na malaki ang impluwensiya ng ibang katutubong wika sa wikang Chabacano sapagkat araw-araw na nakikisalamuha ang tao sa kanyang paligid.

Sa mga datos na nakalap ng mananaliksik ay makikita na malaki ang bilang ng mga impormanteng gumagamit pa rin ng purong Chabacano sa rural at urban area. Nangangahulugan na malaki pa rin ang impluwensiya ng wikang Español sa wikang Chabacano sa lungsod ng Zamboanga. Ilan sa mga halimbawa nito ay ang mga salitang abuela, pierna, fuego, garrote, hombre, jabon, lagrimas, mujer, perro, cuarto, rodillas, antiguo, buenmoso, Hermosa, hermoso, canalla, duru, grueso, flaco, fuerte, corre, mal olor, embustero, engañador, arripintido, garrucha, golpea, pega, supla, embona, vengga at vende. Ito ang mga salitang parehong-pareho ang kahulugan at katumbas sa wikang Español.

Subalit ayon kay Semorlan (2002), may kaibahan ang Chabacano sa Lenguahe ng Espanya, kaya imposibleng maunawaan ng isang Español ang wikang Chabacano. Lumabas sa pag-aaral na ito na ang ilang leksikal na katawagan ay hindi magkatulad ang kahulugan sa wikang Español. Halimbawa nito ay ang salitang Chabacano na empeinera na nangangahulugan na hita sa Filipino ngunit sa wikang Español ito ay nangangahulugang "I started". Ganoon din ang mga salitang pega, supla at embona na ang katumbas nito sa wikang Filipino ay paluin subalit sa wikang Español ito ay nangangahulugan na malagkit para sa salitang pega, "supply" para sa salitang supla at tama ang sukat para sa salitang embona.

Dagdag pa ni Semorlan (2002), nahahaluan ng Tausug, Subanon, Bisaya ang Chabacano sa Zamboanga samantalang nababahiran naman ng Tagalog ang Chabacano sa Cavite. Lumabas din sa pag-aaral na malaki rin ang impluwensiya ng mga nabanggit na mga wika lalong-lalo na sa urban barangay.

Ang mga salitang lumitaw sa ginawang pag-aaral ay ang mga sumusunod: yan sisi, hita, luha, pitaka, tuhod, 
kuripot, guapo, paspas, iru, yan mahay, at maru ay kadalasang impluwensiya ng mga wikang Tagalog, Bisaya, Tausug at Subanen. Ito'y tumutugon sa sinabi ni Cuartocruz (1990) mula kay Santiago (1979), na ang wikang Chabacano ay lumaganap din sa Cavite, Ermita, Ternate at Zamboanga. Ang may pinakamalaking populasyon ng mga taong gumagamit ng wikang ito ay matatagpuan sa lungsod ng Zamboanga, probinsya ng Basilan, maliit na bilang ng mga Tausug, Samal at Yakan na halos lahat ay mga muslim ay gumagamit din ng ganitong wika. Dahil na rin sa patuloy na paglipat na matitirhan ng mga tao sa rural at urban area, malaki ang impluwensiya ang wikang ginagamit sa isang pook. Ang Zamboanga ay isang lungsod sa rehiyon na Tangway ng Pilipinas. Ito ay may kabuuang land area na 1,483.38 sq.km. Ikatlong pinakamalaking lungsod ito ng Pilipinas sa lupain at may kabuuang 98 barangay na nahahati sa rural barangay at urban barangay. Sa pagdating ng ibang mga lahi na tumira sa lungsod ng Zamboanga partikular sa urban barangay, hindi maiiwasang naiimpluwensiyahan ng mga grupong ito ng mga nakapaligid sa kanila.

Ang lungsod ng Zamboanga ay tinatawag na "melting pot" ng iba't ibang kultura. Ito ay binubuo ng iba't ibang grupo ng mga tao na may sariling kultura. Ayon sa 2010 NSO census:

Of the household population in Zamboanga City, 38.4 percent reported ZamboangueñoChavacano as their ethnicity. The other were reported as belonging to these ethnic groups: Tausug (22.2\%), Bisaya/Binisaya (20.7\%), Sama/Samal (4.4\%), Yakan (3.4\%), Sama Bangingi (2.9\%), Hiligaynon/Ilonggo (1.8\%), Tagalog (1.6\%), Cebuano (0.9\%) Subanen (0.8\%), and other. (NSO census, 2010)

Ang mga dahilang ito ay nagpapatunay sa teorya ni Holmes (1992), na ang mga tampok na varyasyon ng wika ay bunga ng demograpikong salik gaya ng heyograpikong pagkakaiba ng mga ispiker. Ayon kay Morano (2002), ang dalawang mahalagang dahilan ng pagkakaroon ng varayti ng wika ay ang (1) heograpikong lokasyon ng komunidad ng pagsasalita (speech community) o kaya'y mga pook kung saan ginagamit ang wika na maaaring nahihiwalay ng isang anyong tubig o kabundukan, at (2) language boundary dulot ng migrasyon o paglilipat ng komunidad sa ibang lugar marahil dala ng isang mapinsalang kalamidad o giyera, o ang unti-unting pagsasama ng dalawang dating magkahiwalay na komunidad. Dahil dito, nagkakaroon ng iba't ibang wika at nasasagkaan ang komunikasyon ng mga tao.

Maliban sa mga dahilang ito, ang wikang Chabacano ay may orihinal na katangiang creole. Ang creole ay isang varayti ng wikang nadebelop mula sa wikang pidgin. Ang pidgin ay nabuo o lumutang mula sa simplipikasyon ng wika kung saan ang wikang iyon ang nagdodomina o nakalalamang mula sa iba pang wika ng iba't ibang tagapagsalita.

Sa Filipinas, ang tanging dumaan sa prosesong creolization ay ang wikang Chabacano ng lungsod Zamboanga. Ang proseso ng creolization ay dumadaan sa alinmang yugto ng pag-unlad ng isang pidgin maaaring gradwal na creolization o biglaang creolization. Ang gradwal na creolization ay mangyayari sa pinahaba o pinalawak na yugto ng pidgin. Sa madaling salita, ang creolization ay nagsisimula sa yugto kung saan ang pidgin ay lubos na nadebelop. Ito ay nailalarawan sa pamamagitan ng pagkakaroon ng pamantayan ng paggamit ng wika. Magaganap naman ang biglaang creolization sa proseso bago lumabas ang matatag na pidgin mula sa maagang pag-unlad nito. Sa puntong ito, ito ay nailalarawan pa rin sa pamamagitan ng paggamit ng wika.

Sa paglipas ng maraming panahon, mula sa ugnay na wika ng mga Kastilang mananakop at wika ng lokal sa lugar ay nagkaroon ng komunidad ng pagsasalita. Napaunlad ang gramatika, lumalawak ang bokabularo at hindi na lamang ito wika ng mananakop bagkus naging bernakular na wika ito ng lungsod ng Zamboanga, (Hufana et al, 2018).

\section{Kongklusyon}

Batay sa mga natuklasan mula sa pag-aaral na ito, ang mga sumusunod ang nabuong kongklusyon. Ang 
wikang Chabacano ay katulad din sa ibang mga wika sa Filipinas na sumasailalim din sa varyasyon tulad ng wikang Filipino. Ang resulta ng pag-aaral ay nagpapatunay sa teorya ni Holmes (1992), na ang mga tampok na varyasyon ng wika ay bunga ng demograpikong salik gaya ng heyograpikong pagkakaiba ng mga ispiker, bagama't may mga salita pa ring nananatili sa rural at urban barangay ang mga datos ay nagpapakita na mas maraming leksikal na salita na nananatiling puro sa rural barangay ng lungsod ng Zamboanga.

Sa sosyolinggwistikong pananaw, Ang pagkakaroon ng varyasyon ng wikang Chabacano ay ang pagkakaroon ng maraming wikang ginagamit sa kani-kanilang lugar na nakapagdudulot ng malaking impluwensiya sa pagkakaroon nila ng iba-ibang salita na kung minsan ay galing sa ibang wikang sinasalita ng ibang komunidad. Ang paggamit ng iba pang wika dahil sa pananahan ng ibang tribo ay siyang malaking kontributor sa pagkakaroon ng varayti at varyasyong leksikal ng wikang Chabacano. Ganoon pa man, masasabi sa pag-aaral na ito na ang mga taong naninirahan sa rural at urban barangay sa lungsod ng Zamboanga na isang taal na Chabacano ay kakikitaan ng varayti. Ang pagkakaiba-iba nila ng mga panumbas sa iisang salita at paraan ng pagkakabuo ng mga salita ay nagpapatunay sa sinabi nina Paz et al. (2003) na hindi homogeneous ang isang wika dahil may iba-iba itong anyo. Dahil sa tuloy-tuloy na interaksyon at pakikipag-usap ng mga miyembro ng isang grupo o kumunidad tulad ng pamilya, mga kaparehong propesyon, at iba pa na nagkakaroon ng mga katangian ang mga salita nila na naiiba sa mga miyembro ng ibang grupo o komunidad. Dala rin ito ng magkaibang interes, kapaligiran at karanasan ng isa't isa.

\subsection{Rekomendasyon}

Batay sa natuklasan sa pag-aaral na ito, Iminumungkahi ng mga mananaliksik ang pagsasagawa ng pag-aaral na sumasaklaw naman sa iba pang bahagi ng pananalita upang mapagtibay ang pag-aaral na isinagawa at maaaring maging batayan sa paglikha ng mga aklat pambalarila at leksikograpiya ng wikang Chabacano. Iminumungkahi sa kaguruan na gumawa ng kaukulang aksyon sa pagbibigay-halaga sa mga wika ng mga mag-aaral bilang batayan sa pagtamo sa layuning pang-edukasyon. Ang mga guro ng wika ay kinakailangang may sapat na kabatiran sa papel ng wika sa edukasyon. Iminumungkahi rin ng mga mananaliksik na magsagawa ng ganitong pag-aaral na nakatuon naman sa iba pang katutubong wika sa lungsod ng Zamboanga.

\section{Mga sanggunian}

Bernales, R. O., Atienza, G. C., Talegon Jr., V. M., Achas, A. P., Danao, L. D., Joson, E. A., Ornos, P. S., Rovira, S. G., \& Suguran, T. S. (2006). Academic Filipino towards effective communication [Akademikong Filipino tungo sa efectivong komunikasyon]. Valenzuela City: Mutya Publishing House Inc.

Cebrero, J. C. (2014). Dayalektal na diversidad ng wikang subanen sa Zamboanga peninsula [Unpublished masteral thesis]. Mati San Miguel: Cerilles Sate College.

De Castro, I. P., Ampil, R. DL., Cabrera Jr., H. I., Marquez, F. F., \& Supnet, A. L. (2007). Filipino language in academic communication [Wikang Filipino sa akademikong komunikasyon]. Valenzuela City: Mutya Publishing House Inc.

Hufana, N. H., Banawa, MJ. D., Gervacio, G. V., Pantorilla, C. R., Sajulga, A. C., \& Tiosen, R. B. (2018). Language and culture in a peaceful society [Wika at kultura sa mapayapang lipunan]. Malabon City: MutyaPublishing House, Inc.

Komisyon sa Wikang Filipino - Panrehiyong Sentro ng Wikang Filipino. (2002). Chabacano-Filipino-English dictionary [Diksyunaryong Chabacano-Filipino-Ingles]. Zamboanga City: PSWF-WMSU.

Lachica. V. S. (1994). Communication and linguistics [Komunikasyon at linggwistika]. GMK.

Paz, C. J., Hernandez, V. V., \& Peneyra, I. U. (2003). The study of language [Ang pag-aaral ng wika]. Quezon City: The University of the Philippines Press.

Peregrino, J. M., Constantino, P. C., \& Ocampo, N. S. (2002). Minanga: Variety readings and variation of Filipino [Minanga: Mga babasahin sa varayti at varyasyon ng Filipino]. Quezon City: SWF-UP.

Philippine Statistics Authority. (2013). About census activities. Retrieved from https://psa.gov.ph/content/population-zamboanga-city-four-times-higher-40-years-results-2010-census- 
Pagsipat sa mga salik na nakaimpluwensiya sa varayti at varyasyong leksikal sa wikang Chabacano population-and

Santos, A. L., Magracia, E. B., \& Semorlan, T. P. (2010). Variety and variation of the Filipino language [Varayti at varyasyon ng wikang Filipino]. Iligan City: Departamento ng Filipino MSU-IIT.

Semorlan, T. P., Semorlan, A. P. Mariño, F. C., \& Fernandez, E. C., (2000). The literature and Filipino culture [Ang panitikan at kulturang Pilipino]. Quezon City: C \& E Publishing, Inc. 
Drapeza, H., \& Reyes, A.

54 Consortia Academia Publishing (A partner of Network of Professional Researchers and Educators) 